Polymer J., Vol. 2, No. 5, pp 560-580, (1971)

\title{
Swelling, Deformation, and Retraction of Poly(4-methylpentene-1). I. Morphological Observations
}

\author{
Yoshimichi Hase* and Phillip. H. GeIL \\ Division of Macromolecular Science, \\ Case Western Reserve University, \\ Cleveland, Ohio 44106, U.S.A.
}

(Received November 9, 1970)

\begin{abstract}
The deformation and retraction of bulk poly(4-methylpentene-1) (P4MP1) in the swollen state was studied by electron microscope and wide angle X-ray diffraction. P4MP1 swollen with various swelling agents can be stretched several hundred percent at room temperature in a macroscopically uniform manner. On the microscopic level the deformation is quite heterogeneous. If allowed to relax, a certain fraction of the deformation is recovered instantaneously followed by further recovery with time. Repeated replica electron micrographs of the same area indicate that pulled-out fibrils take a major role in the retraction at high elongation and that interlamellar slip is predominant at low elongation. Use of a solid swelling agent permitted replication of the swollen polymer; swelling occurs by separation of lamellae revealing interlamellar links which retract upon deswelling.

KEY WORDS Poly(4-methylpentene-1) / Swelling / Deformation /

Retraction/Electron Microscopy/X-ray Diffraction/Separation of

Lamellae /
\end{abstract}

Although some features of the deformation process of crystalline polymers appear to be understood at present, very little is known of the structure of the drawn region or the mechanism of inhomogeneous deformation. Also the mechanism of shrinkage of drawn polymer upon relaxation or heat-treatment is far from being understood despite its industrial importance; e.g., processing of crimped yarns by means of a combination of inhomogeneous deformation and relaxation and/or heat-treatment. In addition the nature of swelling of crystalline polymers has not been examined in detail. In this paper the swelling, deformation, and retraction of bulk poly(4-methylpentene-1) (P4MP1) were studied by means of electronmicroscopy and wide-angle $\mathrm{X}$-ray diffraction (WAXD).

Melt-crystallized P4MP1 films, under crystallization conditions yielding a well-developed lamellar structure, are quite brittle even at elevated temperature unless they are swollen with various swelling agents. When swollen, deformation up to several hundred percent can be

* Present address: Teijin Limited, Plastic Reseach Laboratory, 37-19, 3-chome, Oyama, Sagamihara, Kanagawa, Japan. obtained at room temperature. Although it is macroscopically uniform it is quite heterogeneous on the microscopic level. In addition a certain amount of retraction of the drawn region was obtained upon releasing the tension. Similar effects, i.e., large increase in ductility and a fairly large amount of retraction, were previously reported by Hay and Keller $^{1}$ for polyethylene (PE) when moistened with a swelling agent; however nothing is known about the mechanism. Because of the heterogeneous nature of the deformation and multi-component surface morphology of the drawn region, it was necessary to observe the same selected area before and after retraction in order to determine which component was playing the major role in the retraction. Systematic examination of the morphological change during the retraction process was carried out using repeated replicas of the same area; these clearly showed that shrinkage of the pulled-out fibrils was predominant at high elongation while interlamellar slip took place at low elongation.

In the lamellar model for crystalline polymers one would expect swelling to occur in the interlamellar regions, the amount of swelling depending on the numbers and length of various inter- 
lamellar links and tie molecules. In this work we also examined the nature of the swelling process. Electron microscopic studies enabled us to observe the separation of the lamellae and revealed fibrillar links between the lamellae whose behavior upon deformation and retraction could be followed; although some evidence of apparently similar interlamellar links has previously been reported ${ }^{2,3}$ almost nothing is known of their properties. Upon deswelling these firillar links retracted, presumably in a similar manner as those produced by large scale drawing.

\section{EXPERIMENTAL}

\section{Sample Preparation}

P4MP1 (TPX polymer*, Grade; RTM/natural) was compression-molded into either $1-\mathrm{mm}$ or 3-mm thick sheets at $274^{\circ} \mathrm{C}$. The 3-mm thick sheets were primarily used for fracture experiments and the 1-mm thick ones for the deformation experiments. For high-crystallinity specimens, the molded sheets were slowly cooled under pressure from the molding temperature to room temperature by turning off the heater of the press. For low-crystallinity specimens, the molded specimens were rapidly quenched from the molding temperature by immersion in ice water.

In order to get a free surface for electron microscopy, the molded specimens were again placed, for less than a minute, between the hot plates $\left(274^{\circ} \mathrm{C}\right)$ of the molding apparatus without contacting the upper plate. After the specimen surface was observed to be slightly remelted, they were taken out, placed on a bench and allowed to cool at room temperature.

\section{Electron Microscopy}

A Hitachi HU-11A electron microscope was used for all the electron microscopic work described in this paper. A conventional platinum - carbon/poly(acrylic acid) replication technique was applied in all cases for which it was applicable. Frequently it was found that some of the P4MP1 remained attached to the replica when it was stripped. Therefore, for repeated replicas of the same area, collodion or gelatin two-stage replication techniques were applied

\footnotetext{
* Trade mark of Imperial Chemical Industries.
}

in spite of their rather poor resolution.

For repeated replication of the free surface before and after retraction, the collodion twostage replication technique was applied to the area of interest marked with a scratch pattern (double cross, $c a .1 .5 \mathrm{~mm} \times 1.5 \mathrm{~mm}$ ).

Neither the conventional or the collodion replication technique could be applied when repeated replicas from bromo-chlorobenzene (BCB, the solid swelling agent used) swollen specimens were needed; in this case the gelatin replication technique was applied in spite of its complexity and poor resolution. Collodion could not be used since isoamyl acetate, the solvent for collodion, dissolves $\mathrm{BCB}$; deswelling occurs during the first replication process if the collodion solution is applied to the BCB swollen specimen. The procedures are essentially the same as for the collodion method except that a backing of collodion was used to avoid breakage of the carbon replica due to swelling of the gelatin.

\section{RESULTS}

\section{Swelling and Swelling Agents}

Diffusion of low-molecular-weight materials, such as swelling agents or plasticizers in polymer solids, is a subject of concern not only for elucidating the segmental motion of polymer molecules but also because of its industrial interest in connection with such properties as gas permeability and dyeability. It has been studied extensively in the past ${ }^{4}$.

In this work, more stress was placed on elucidating the structure of the swollen state and the deformation behavior in the swollen state rather than examining the sorption mechanism. Primarily liquid swelling agents were used for the deformation study, with swelling agents which are solid at room temperature being used for studying the polymer structure in the swollen state. Using a solid swelling agent has a big advantage for examinations of the internal structure in the swollen state; the swollen specimen at room temperature can retain all of the absorbed swelling agent for sufficiently long time, retaining the specimen shape, that replicas of the fractured swollen specimen can be prepared by a conventional replication technique. If 
liquid swelling agents were used a complicated freeze replication would be needed. Similar solid swelling agents have been used by DescampsCarlier, et $a l .^{5}$, for their studies of polyethylene spherulites; biphenyl was crystallized between the lamellae. The location and orientation of the biphenyl was detected by X-ray diffraction at wide and small angles.

P4MP1 can be swollen, even at room temperature, with a number of chemicals. Table I shows the equilibrium degree of swelling at

Table I. Various chemicals effective for swelling P4MP1

\begin{tabular}{lcc}
\hline Chemicals & $\begin{array}{c}\text { Degree of swelling } \\
\text { (volume } \\
\text { increase, } \%)\end{array}$ & $\begin{array}{c}\text { Amount } \\
\text { extracted, } \\
\text { wt \% }\end{array}$ \\
\hline Benzene & 21.6 & 0.63 \\
Toluene & 26.3 & 0.72 \\
Xylene & 24.2 & 0.52 \\
Carbon tetrachloride & 70.7 & 2.4 \\
Methylene dichloride & 20.0 & 0.65 \\
1-Chlorobutane & 33.4 & 0.93 \\
1-Chlorohexane & 25.9 & 0.59 \\
1-Chlorooctane & 19.7 & 0.42 \\
1-Chlorodecane & 16.3 & 0.27 \\
\hline
\end{tabular}

a The waxy material extracted by various swelling agents at room temperature showed a very low melting point, $35^{\circ} \mathrm{C}$, but its WAXD pattern was almost the same as that of the polymer itself except for a slight diffuseness of the diffraction rings.

room temperature for 1-mm thick quenched samples for several liquids. In most cases equilibrium was obtained after several hours immersion. The degree of swelling as a function of time and temperature was estimated from the weight gain of the specimens using the following equation.

$$
\text { Degree of swelling }=\frac{W_{2}-W_{1}}{W_{1}} \times \frac{\rho_{1}}{\rho_{2}} \times 100(\%)
$$

where $W_{1}$ and $W_{2}$ are the weight of the original and the swollen specimen respectively, and $\rho_{1}$ and $\rho_{2}$ are the density of the polymer and that of the swelling agent respectively.

Bromo-chlorobenzene (BCB), biphenyl, bromobiphenyl, bromonaphtol, bromo-hydroxybenzene, carbon tetrabromide, and dichloro-nitrobenzene, all of which are solid at room temperature but melt below $100^{\circ} \mathrm{C}$, were investigated. Among these swelling agents, $\mathrm{BCB}$, whose melting point is $67^{\circ} \mathrm{C}$ and which can swell P4MP1 up to $50 \%$ by volume at $140^{\circ} \mathrm{C}$ within several hours, turned out to be the best; the degree of swelling could be easily controlled by changing the swelling temperature and sufficient swelling could be obtained at relatively low temperature where no appreciable degradation or dissolving of the polymer occurred. The other materials either degraded the polymer at temperatures where significant swelling occurred, changing the color to dark brown or black, (bromonaphthol, bromohydroxybenzene and carbon tetrabromide) or only slightly swelled the polymer (biphenyl at $90^{\circ} \mathrm{C}, 6.5$ vol $\%$ ).

It should be mentioned here that when the solid swelling agents are used the cooling rate of the swollen specimen from the high swelling temperature is critical in obtaining the desired degree of swelling and to obtain reproducibility of the data. The swollen specimen must be cooled rapidly to room temperature in order to retain the swelling agent inside the bulk specimen; if cooled slowly the agent is almost entirely exuded during the cooling process. Therefore, in subsequent experiments, when a solid swelling agent was needed, the specimens were swollen with $\mathrm{BCB}$ at different temperatures to yield varying degree of swelling and cooled down rapidly to room temperature; the results are summarized in Table II.

Table II. Swelling of P4MP1a with p-bromo-chlorobenzene

\begin{tabular}{cc}
\hline $\begin{array}{c}\text { Swelling } \\
\text { temp, }{ }^{\circ} \mathrm{C}\end{array}$ & $\begin{array}{c}\text { Degree of swelling } \\
\text { (volume increase, \%) }\end{array}$ \\
\hline 70 & 17.1 \\
90 & 21.3 \\
100 & 24.9 \\
110 & 28.6 \\
120 & 33.1 \\
130 & 43.7 \\
140 & $50.0^{\mathrm{b}}$ \\
\hline
\end{tabular}

a 1-mm thick quenched specimen swollen for $22 \mathrm{hr}$ at a given temperature.

b Partial dissolving of the polymer was observed.

Degree of Swelling

When a solid swelling agent was used, the 
weight of the specimen was measured immediately after removing the sample from the swelling bath, wiping off the excess swelling agent and cooling it rapidly down to room temperature. When liquid swelling agents were used, the excess swelling agent was wiped off and the swollen sample was kept in air for $30 \mathrm{sec}$ before it was weighed. The evaporation rate of the agents on the surface was sufficiently rapid that in most cases the weight remained essentially constant after $30 \mathrm{sec}$.

Absorption of benzene is plotted in Figure 1 as

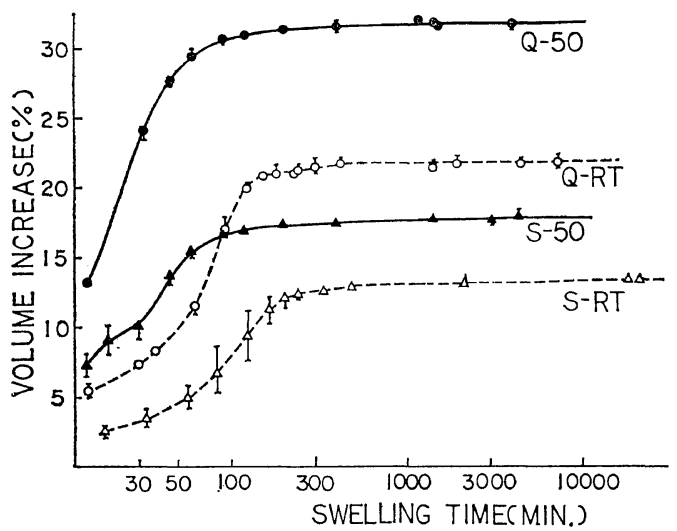

Figure 1. Absorption of benzene at room temperature ( $\mathrm{RT}$ ) and $50^{\circ} \mathrm{C}$ (50) in $\mathrm{P} 4 \mathrm{MP} 1$ compressionmolded, 1-mm thick films, quenched (Q), and slowly crystallized (S), respectively.

a function of swelling time. Half time for the quenched and slow-cooled specimens at room temperature is 57 and $83 \mathrm{~min}$, respectively, at $50^{\circ} \mathrm{C}$ it is about $19 \mathrm{~min}$ for both cases. As would be expected since the measurements were made at temperatures near $T_{\mathrm{g}}$ of the polymer $\left(T_{\mathrm{g}}=32^{\circ} \mathrm{C}^{6}, 18-29^{\circ} \mathrm{C}^{7}\right)$, sorption showed non-
Fickian behavior.

\section{Reversibility of Swelling}

Except for the fact that a small amount of low-molecular-weight material is extracted from the swollen specimen, as shown in Table $I$, the swelling of this polymer with various solvents appears completely reversible in that (1) the dimensions before swelling and after deswelling are the same, (2) the WAXD patterns before and after swelling show no difference, and (3) the melting points measured by DTA $\left(10^{\circ} \mathrm{C} / \mathrm{min}\right.$, heating rate) were the same in the original specimen and the one swollen and deswollen completely, $241^{\circ} \mathrm{C}$. Figure 2 shows the WAXD patterns of 1-mm thick P4MP1 specimens taken during the swelling-deswelling process; these patterns show that the swelling agent does not penetrate the crystalline region. The pattern suggests that the agent is effective only in the amorphous or inter-lamellar regions and consequently leaves no significant change in properties after being removed. The similarity of the WAXD patterns shown here was confirmed by diffractometry which indicated no shift of peak positions nor any broadening of the peaks.

In addition to the above similarities, the surface morphology of the original specimen was quite similar to that of the benzene-swollen and dried specimen. Figure $3 \mathrm{a}$ is an electron micrograph of the free surface of the original specimen and Figure $3 b$ is the free surface of $a$ sample which had been fully swollen with benzene and later deswollen by evaporating the benzene completely. The most predominant structures in both cases are the flat lamellae and the rod-like structures which may be the edges of lamellae at an angle to the surface. However it should be pointed out that they are

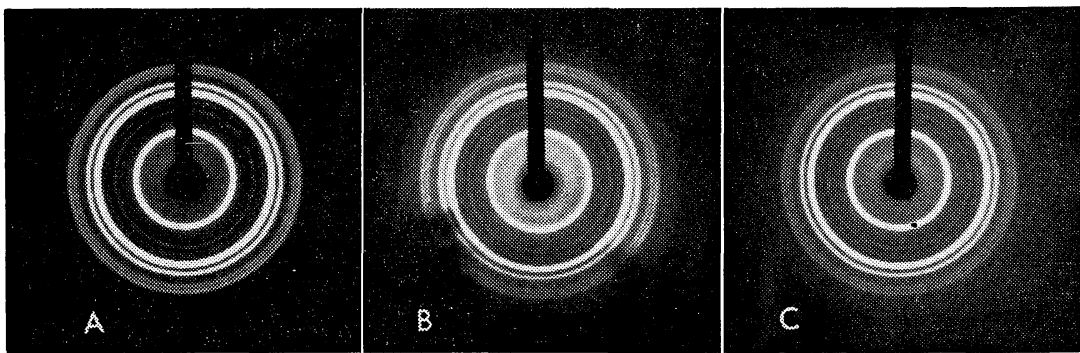

Figure 2. WAXD patterns of original (A), in the swollen state (B), and after deswelling (C) of molded P4MP1. Swelling with benzene at room temperature. 


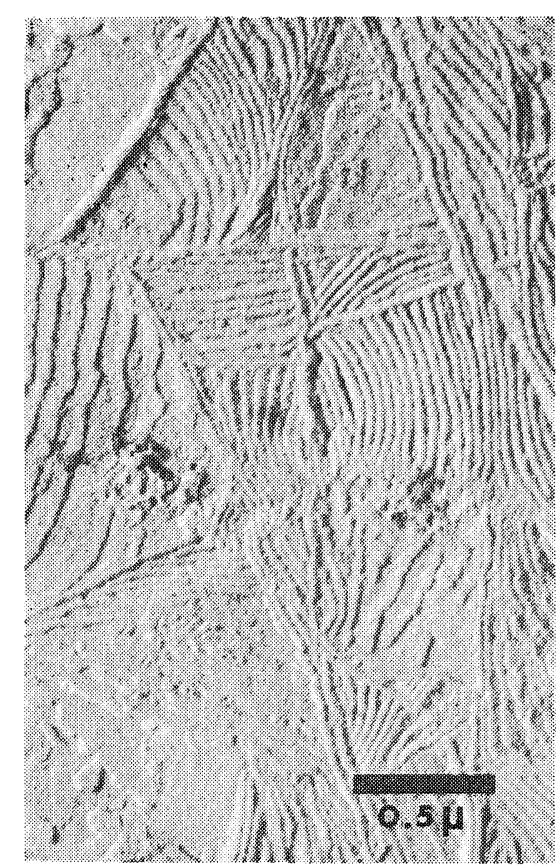

(a)

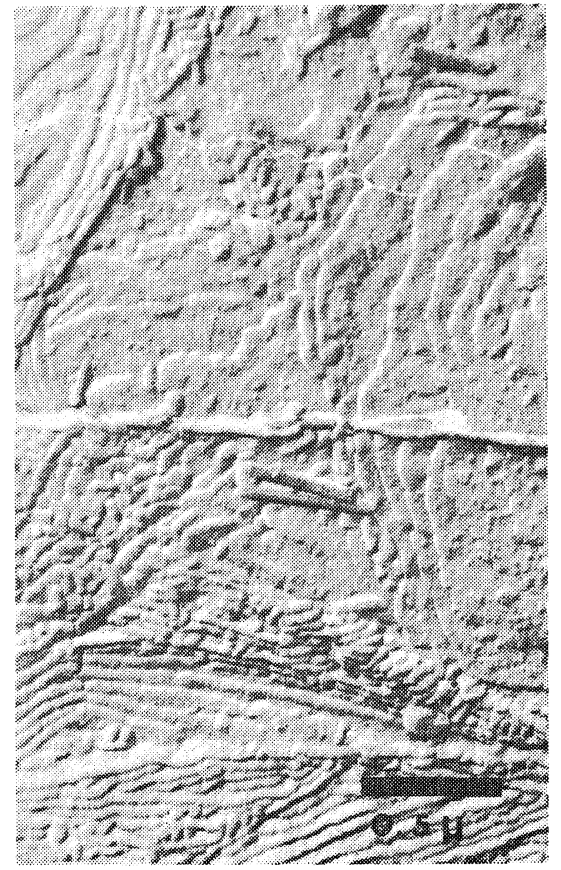

(b)

Figure 3. Electron micrographs of a free surface of a molded P4MP1 (a) and one (b) which had first been fully swollen with benzene and then deswollen completely.

thicker than the step height of the lamellae parallel to the surface. Some short rod-like structures or bumps are often observed on the deswollen surface. At present we have not been able to characterize this material and do not know whether it is segregated low-molecularweight material or fragments of the original rodlike structures. Extraction of the polymer pellets with benzene using a Soxhlet extractor indicated that a certain portion of the polymer consisted of a waxy material, ca. $1.5 \%$ for a $40^{\circ} \mathrm{C}$ extraction and $4-5 \%$ for a $70^{\circ} \mathrm{C}$ extraction, whose melting point was considerably lower than that of the polymer (about 40 and about $150^{\circ} \mathrm{C}$ for the 40 and $70^{\circ} \mathrm{C}$ extraction respectively) but whose WAXD pattern was almost the same as that of the polymer itself except for a slight diffuseness of the diffraction rings. The rod-like structures are also observed on the surface of highly stretched films, shown later on, suggesting that they are one of the fundamental structures of the polymer and mayn ot be merely lamellae on edge.
The reversible characteristics of the swelling were also observed in the mechanical properties measured as a function of degree of swelling (subsequent paper $^{8}$ ). When the mechanical properties of the swollen polymer, such as initial modulus, yield stress, and elongation at yield, were measured while the degree of swelling was decreasing from the fully swollen state, these properties extrapolated directly toward the values of the original untreated specimen.

From these experiments it can be concluded that the swelling of P4MP1 is quite reversible, and consequently for the deformation and retraction studies in the swollen state discussed below, it is assumed that a sample swollen to a given amount is in the same physical state regardless of whether it was swollen to a great extent and then partially dried or only partially swollen initially.

\section{Localized Deformation Caused by Swelling}

Since the WAXD data strongly suggests that the swelling agent is located between the lamellae, it is of interest to directly observe the poly- 
mer structure in the swollen state. The 3-mm thick quenched or slowly crystallized specimen

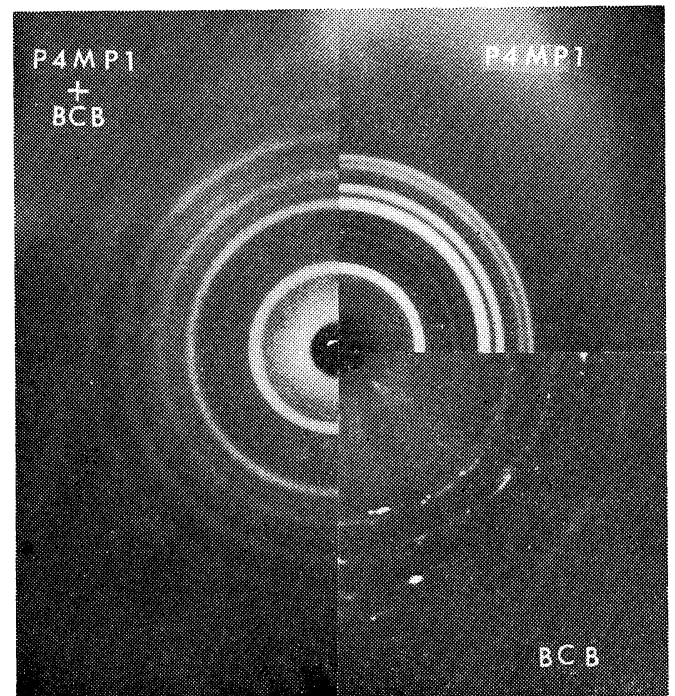

Figure 4. WAXD patterns of molded P4MP1 (upper right), powder of $\mathrm{BCB}$ (lower right), and molded P4MP1 swollen with BCB (left).

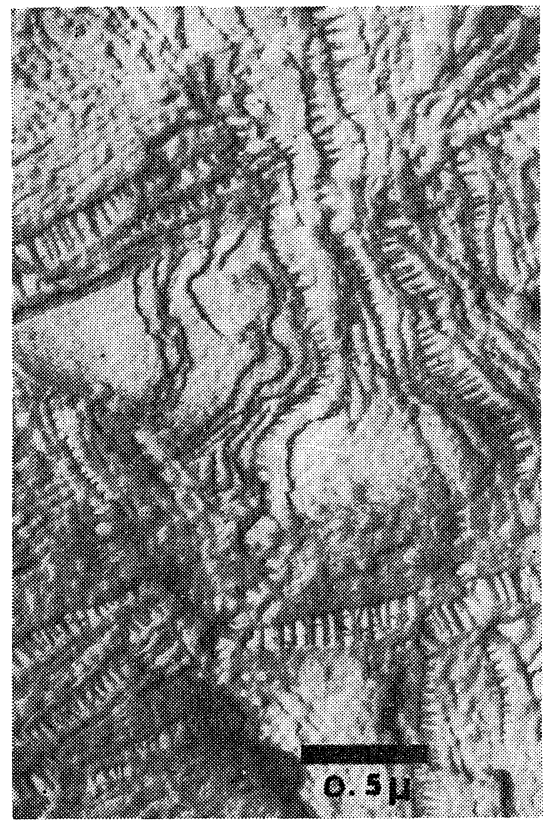

( a ) was immersed in molten $\mathrm{BCB}$ at $100^{\circ} \mathrm{C}$ for about $50 \mathrm{hr}$, yielding 22 and $15 \%$ swelling respectively. The polymer specimen, originally almost transparent, looked semitransparent in the molten swelling agent but turned opaque as it cooled down to room temperature and the swelling agent crystallized. A WAXD pattern of this specimen, an $c a .0 .5-\mathrm{mm}$ thick slice being cut out from the bulk, showed two sets of DebyeScherrer rings corresponding to both the P4MP1 and the swelling agent (Figure 4). Although the swelling agent sublimes rapidly at room temperature if it is kept free, it was retained in the bulk specimen for sufficiently long time for our experiments.

After the swollen specimens were cooled to room temperature they were fractured in liquid nitrogen and replicated at room temperature. Presumably the BCB near the fracture surface sublimed before replication. Fracture surface photographs of the slowly crystallized specimen and the quenched specimen are shown in Figure $5 \mathrm{a}$ and $\mathrm{b}$ respectively. In the quenched speci-

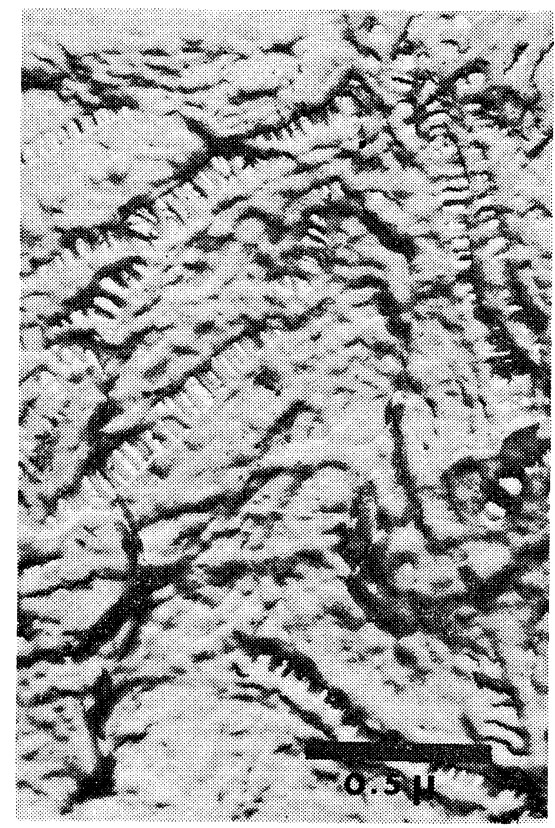

( b )

Figure 5. Electron micrographs of a fracture surface of slowly crystallized (a) and quenched (b) 3-mm thick P4MP1 swollen with BCB. Pt-C replica. The specimens were swollen at $100^{\circ} \mathrm{C}$ and gradually cooled down to room temperature before fracturing at liquid nitrogen temperature. 
men the structure is not so well developed and the direction of lamellar alignment is difficult to determine. However it is easily seen that the cracks in the slowly crystallized specimen are oriented parallel to the lamellae. Numerous fibers connect opposite surfaces of cracks having various widths and lengths.

It should be pointed out that at the beginning of the experiment there was some uncertainty concerning the definition of the parallel lines as lamella edges; they are very thick (ca. $400 \AA$ ) compared to the lamellae observed parallel to

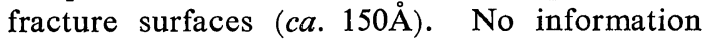
about the molecular orientation in the "lamellae" on the fracture surfaces has been obtained. However they cannot be considered as resulting from lamellae at an angle to the surface because they are so uniform in thickness and so well aligned over wide areas. Also pairs of stereo pictures clearly revealed that they are the edges of the lamellae fractured normal to the plates leaving no question about these parallel line structures.

Fracture appears to occur most readily perpendicular or parallel to the lamellar surface. A saw-tooth fracture surface of unknown origin (regions $F$ and $G$ in Figure 8) is occasionally produced in regions where the lamellae are at a small angle to the normal to the surface. In only a few regions, however, is there an apparent transition to surfaces on which greater and greater extents of lamellar surfaces are exposed as the lamellae lie at larger angles; rather only large areas of lamellar surface are seen as near region $E$ in Figure $8 \mathrm{c}$ and Figure $5 \mathrm{a}$. The parallel orientation of the cracks and the lamellae was observed throughout the sample. It should also be emphasized that no cracks were observed in the region where the lamellae are lying parallel to the fracture surface. The fracture surface of an untreated sample looks exactly the same except that there are no cracks and connecting fibrils.

The cracks could be found over the entire fracture surfaces but they were quite inhomogeneous in size and distribution. This suggests that either the swelling agent is quite heterogeneously distributed from the beginning or it can migrate from an original uniformly distributed state during its crystallization yielding the inhomogeneously distributed cracks. In order to determine this point, the swollen specimen was quenched from the swelling temperature, $100^{\circ} \mathrm{C}$, to liquid nitrogen temperature in the hope of restricting the possible migration of the swelling agent. The fracture surface of this specimen is shown in Figure 6; it shows more

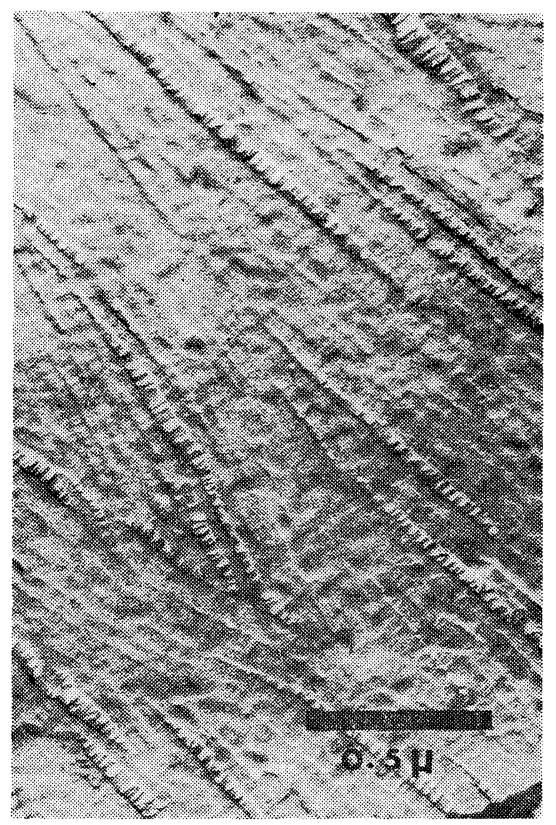

Figure 6. Electron micrograph of a fracture surface of slowly crystallized P4MP1 swollen with BCB. The swollen specimen was quenched from $100^{\circ} \mathrm{C}$ into liquid nitrogen. Pt-C replica, perpendicular shadowing.

evenly separated lamellae with narrower and more uniformly distributed cracks. As mentioned previously the swelling agent can be almost completely squeezed out if the swollen specimen is cooled down slowly to room temperature. Thus there is no doubt that the swelling agent can migrate quite easily during its crystallization process. It thus appears reasonable to assume that the swelling agent is rather uniformly distributed between the lamellae at high temperature and that the observed cracks and fibrils form as the BCB crystallizes. Presumably the interlamellar cohesive strength varies somewhat and the $\mathrm{BCB}$ aggregates during crystallization in the weaker regions.

In order to see the structure of the cracks 


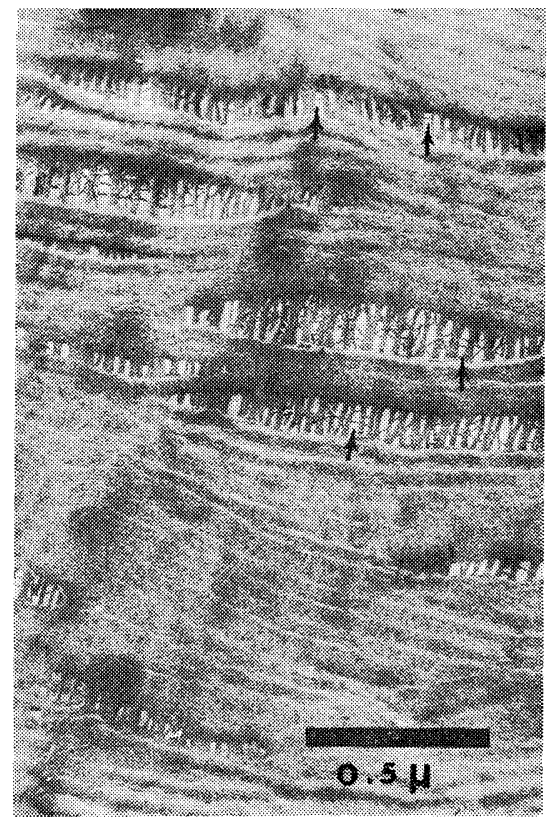

Figure 7. Electron micrograph of a fracture surface of a slowly crystallized film swollen with BCB. Pt-C replica with perpendicular shadowing. Thin fibers lying at a right angle to thick ones are marked by arrows.

and the fibrils in more detail, the fracture surfaces were shadowed from directly above. The perpendicular shadowed fracture surface of the same specimen as in Figure $5 \mathrm{a}$ is shown in Figure 7 under higher magnification. It very clearly reveals two different kinds of fibrils, thick and thin, in the cracks and the parallelism of the cracks and the lamellae. The thick fibrils are aligned almost parallel to each other and connect the opposite side of the crack whereas the thin fibrils lie at an angle between the thick fibrils. In small cracks few thin fibrils are observed but in bigger ones both thin and thick fibrils are seen. The thicker ones show no substantial thinning toward the center except for an apparent branching. We point out here that these fracture surfaces, observed in the swollen state, are the structure one would expect to see if interlamellar links such as originally proposed by Reneker (see ref 3) exist.

If these swollen specimens are dipped in benzene at room temperature and then dried, the swelling agents can be completely removed, resulting in deswelling. Upon deswelling the specimen recovers its original dimension and becomes transparent again. Figure 8 shows the morphological change in a selected fracture surface before and after deswelling. Since the gelatin replication technique was applied, as explained in the experimental section, the resolution is not as good as that in the previous photographs; one can barely see individual fibrils in the cracks. However it is quite obvious that only the fibrils retracted and no change occurred in the other regions. The extent of retraction is not uniform; e.g., the cracks in region $\mathrm{A}, \mathrm{B}$, and $\mathrm{C}$ healed almost completely while the ones in region $\mathrm{D}$ and $\mathrm{E}$ did not. Although it was impossible to compare the thickness of the same specific fibrils before and after retraction, because of the poor resolution of the repeated replicas, an attempt was made to observe the thickness change by measuring the diameter of the fibrils in micrographs specially prepared for this purpose; i.e., photographs taken under high magnification from the perpendicular shadowed $\mathrm{Pt}-\mathrm{C}$ replicas. The average fibril diameter as well as the distribution of fibril diameters are shown in Figure 9 for a slowly crystallized specimen. The data indicate that the average diameter of the thick fibrils as well as the distribution are shifted after deswelling toward higher values by $10-20 \%$. In the case of thin fibrils, the distribution becomes broader, with about a 10-\% higher average value.

\section{Deformation and Retraction Phenomena}

For our microscopy observations, the stretching was carried out in air, using a manually operated stretching device, just after the swollen specimen was taken out of the swelling agent bath. Stretching of the fully swollen specimen in benzene resulted in a much lower elongation at break, at most $200 \%$.

Macroscopically the swollen specimen, when drawn either in air or in a swelling agent, undergoes uniform deformation. However, on a microscopic level, the deformation turned out to be heterogeneous in nature, i.e., micronecking occurred.

The drawn specimens, unless they are dried taut, snap back when they are released. This retraction in the drawn specimens can be brought about by several procedures itemized below. 


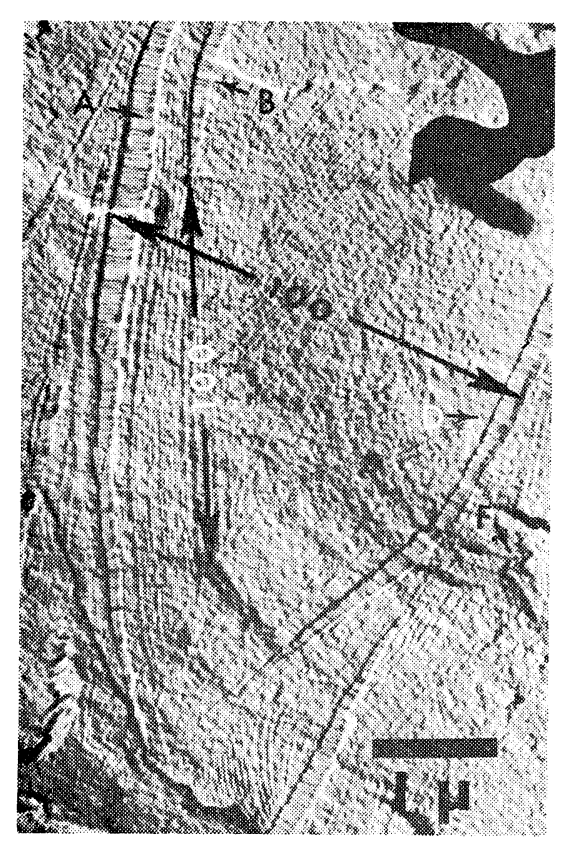

(a)

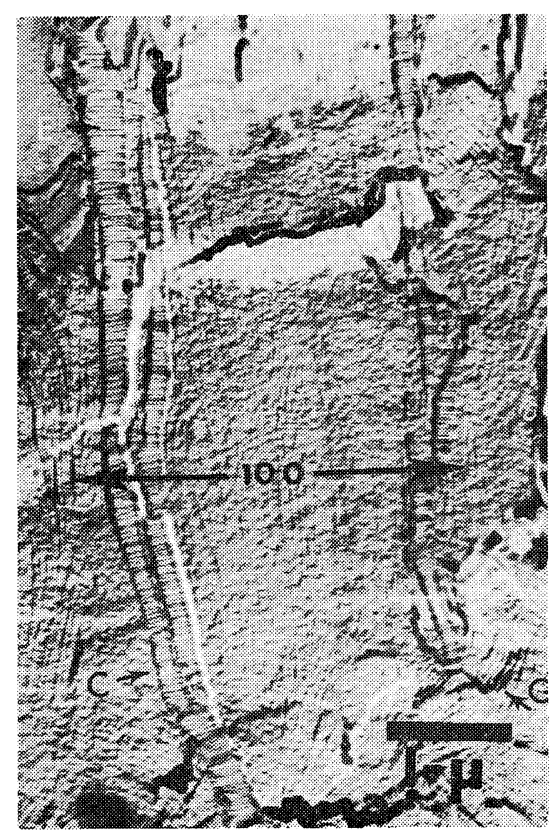

(c)

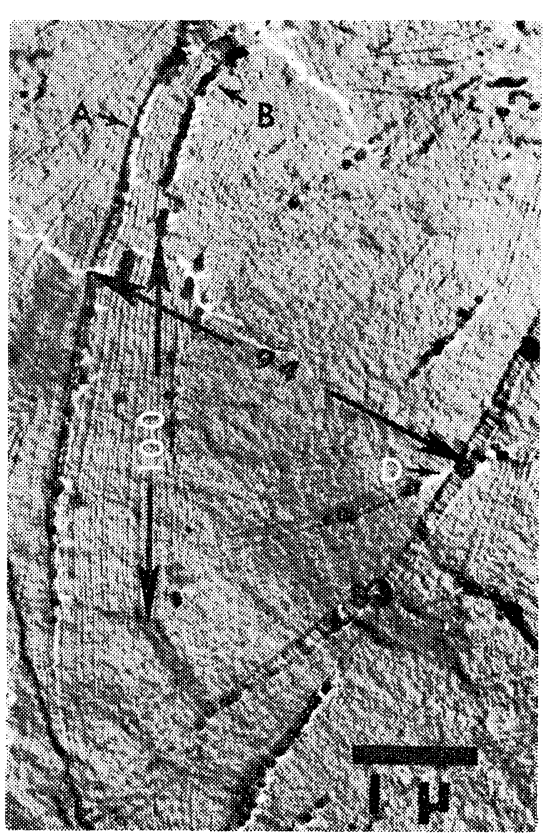

( b )

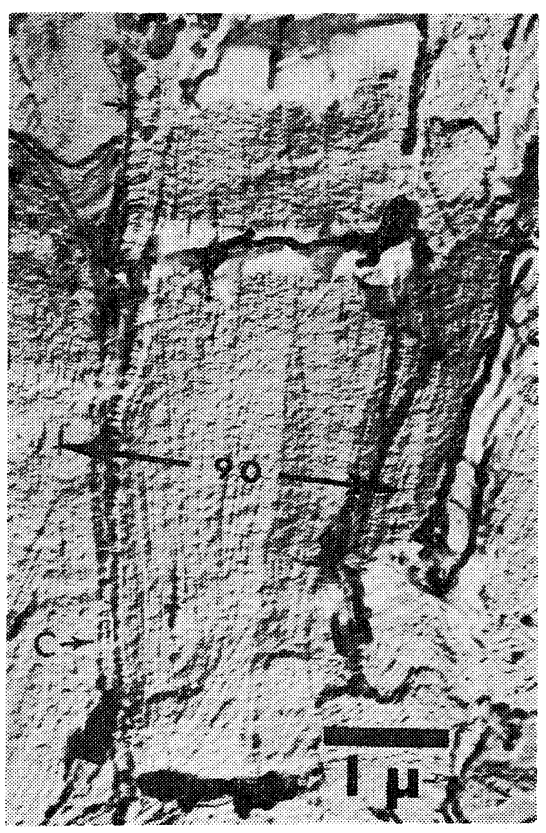

(d)

Figure 8. Electron micrographs of the fracture surface of the slowly crystallized film ((a) and (c)) and after ((b) and (d)) deswelling. Gelatin 2-stage replicas. The distance between the pairs of points is shown in arbitrary units. 


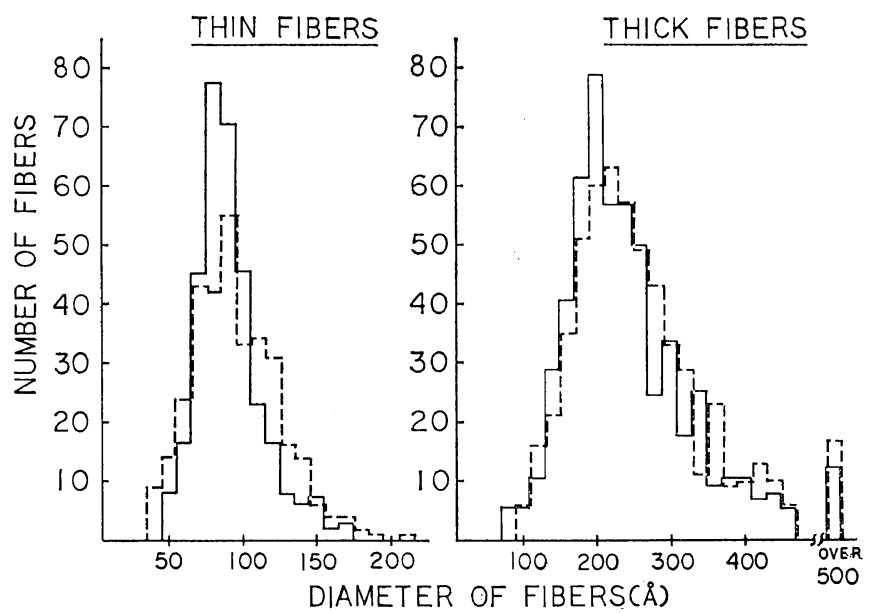

Figure 9. Histograms of the diameter of thick (right) and thin fibers (left) observed on fracture surfaces of slowly crystallized specimens befores (solid line) and after (dotted line) deswelling. Total number of counts of thick and thin fibers were 484 and 342 in the swollen state and 563 and 332 after deswelling respectively. For the graphs the numbers of counts after deswelling were normalized with respect to the ones in the swollen state.

(1) Free drying-the film is released after stretching. Retraction occurs in two steps as the solvent is evaporating; an instantaneous retraction is followed by additional retraction during the drying process.

(2) Reswelling-the specimen which was stretched and dried taut is replaced in the swelling agent with free ends. Retraction occurs in the swelling agent, increasing with time to an equilibrium value, and further retraction occurs during the drying process after the sample is removed.

(3) Heat-treatment-the specimen, which was stretched and dried taut, is annealed with free ends; almost instantaneous retraction to the maximum value occurs at temperatures above $140^{\circ} \mathrm{C}$.

(4) Stress relaxed-the specimens were stretched in air and stored under tension in the swelling agent for a few days. It still snapped back when released, retracting further with time.

Retraction by any of the methods mentioned above occurs at all draw ratios. The relationship between shrinkage and elongation is plotted in Figure 10 for the free dried sample; the ordinate is the percentage of retraction with respect to the stretched length:

$$
\begin{aligned}
& \text { Retraction, } \%=\frac{l_{\mathrm{s}}-l_{\mathrm{r}}}{l_{\mathrm{s}}} \times 100 \\
& \text { Stretched, } \%=\frac{l_{\mathrm{s}}-l_{\mathrm{o}}}{l_{\mathrm{o}}} \times 100
\end{aligned}
$$

$l_{0}$, the original length

$l_{\mathrm{s}}$, the length after the specimen is stretched

$l_{r}$, the length after the specimen retracts

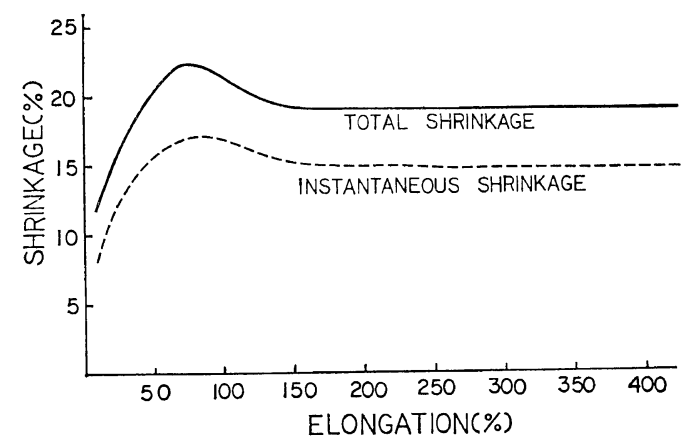

Figure 10. Shrinkage of benzene swollen and stretched P4MP1 films upon free drying as a function of elongation; instantaneous shrinkage (dotted line) and final shrinkage (solid line). 
The dotted line indicates the amount of retraction just after releasing the stretched films, while the solid line indicates the final retraction when dry. The difference between the dotted line and the solid line is the retraction during the drying process. In both cases, the relative percentage of retraction stays constant above $130-\%$ elongation. This means that the amount of shrinkable material increases directly with the amount of elongation after the initial stages of deformation. On the basis of our electron micrographs (see Figures 18 and 19), this result suggests that the shrinkable portion within the drawn specimen is related to the amount of fibrils or material in the microneck regions. The retraction of the pulled-out fibrils is clearly demonstrated in repeated replicas described below.

The retraction caused by the free-drying method is a time dependent phenomenon. In Figure 11 the amount of retraction is plotted as

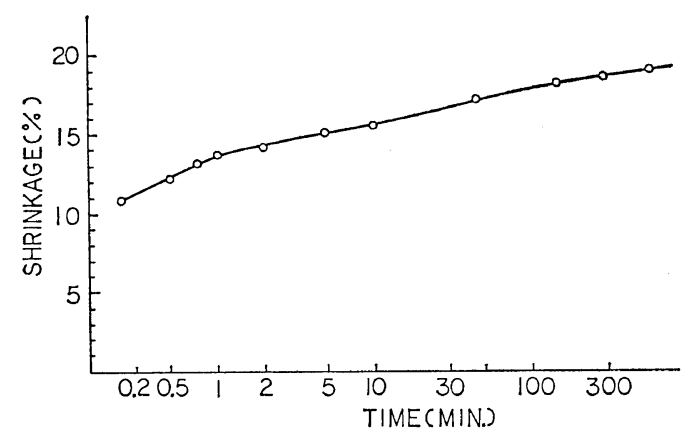

Figure 11. Time dependence of shrinkage upon free drying.

a function of the time elapsed after the stretched film is released and allowed to dry freely. An apparently similar 2 step retraction occurs if the drawn sample is released after being reinserted in benzene. Although data within $5 \mathrm{sec}$ were not obtainable because the retraction was too fast to follow, extrapolation to zero time gives an idea of the portion of the sample which works as an ideal spring. This instantaneous shrinkage (about 10\%) is too large to attribute solely to the expansion of the helical $7_{2}$ conformation of P4MP1 ${ }^{9}$. No difference was observed in the X-ray diffraction patterns, using either photographs or a diffractometer, for sam- ples under tension and dried.

For comparison, the retraction caused by the other methods are shown in Figure 12 as a func-

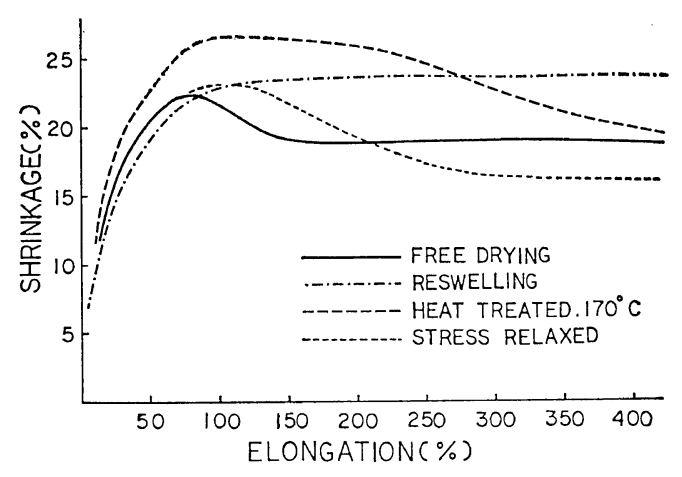

Figure 12. Shrinkage of benzene swollen and stretched P4MP1 films caused by various methods as a function of elongation.

tion of elongation. Up to about $80-\%$ elongation, the amount of retraction of both the reswollen film and the free-dried film are almost the same; above 100-\% elongation the reswollen film retracts more than the free-dried film. The reason for this difference may be attributed to the higher mobility of the oriented molecules in the reswollen polymer as compared with these on the surface of the partially dry sample. The most striking and unexpected result is that the stretched film which had been kept under tension in the swelling agent could still retract nearly the same amount as the other samples. This means that the permanent springs which exist in the stretched film are not relaxed by keeping them in the swelling agent; there was no relaxation of the internal stress giving rise to either the instantaneous or delayed retraction during storage in the swelling agent.

\section{Surface Morphology and WAXD as a Function of Deformation}

The surface replicas of the drawn and dried taut specimen are shown in Figure $13 \mathrm{a}, \mathrm{b}$, and c. At any elongation the swollen specimen showed micronecking. As it is stretched more, the fibril structure becomes more predominant. Even at the highest obtainable elongation (450\%, see Figure $13 \mathrm{c}$ ) there are still considerable numbers of fragments of the original lamellae (marked B), short rod-like structure (marked C) and 
Morphology and Deformation of Swollen P4MP1. I.

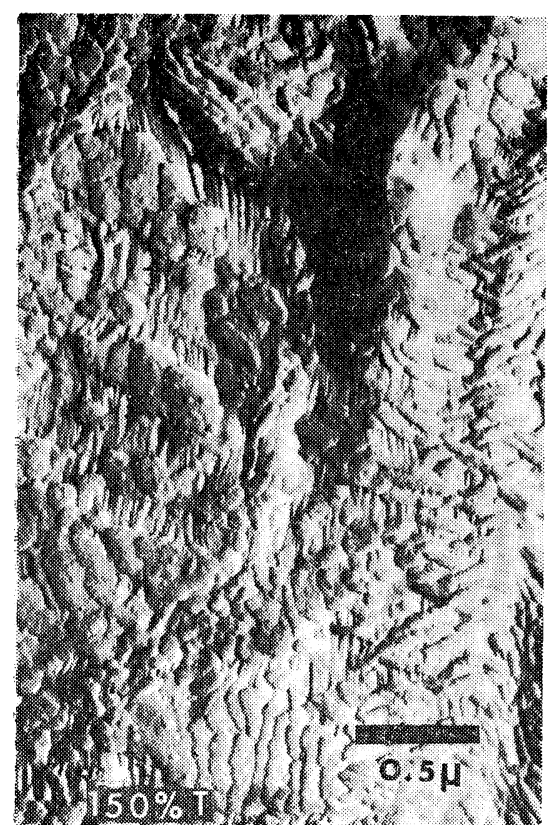

(a)

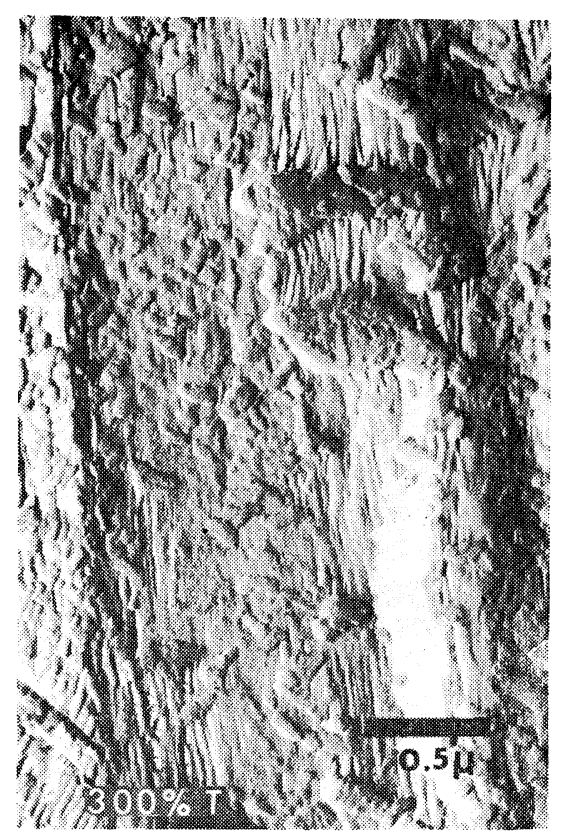

( b )

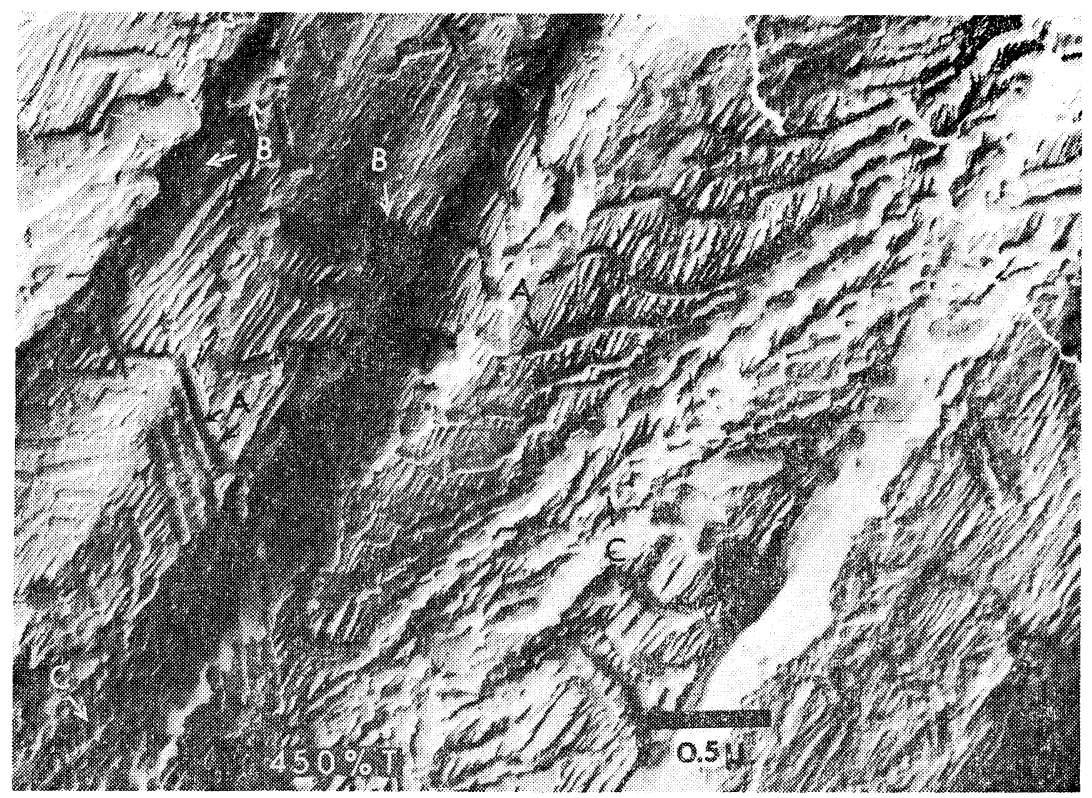

(c)

Figure 13. Electron micrographs of benzene swollen P4MP1 films that were stretched $150 \%$ (a), 300\% (b), and 450\% (c) respectively and dried taut. Long rod-like structures (A), lamellar fragments (B), and short rod-like structures (C) as well as fibers are clearly seen. Pt-C replica. 
long rod-like structure (marked A) connected by "thick" pulled-out fibrils. Thin fibrils are seen more or less perpendicular to the thick one and extending between them. This region resembles very much the fibril structure of the fracture surface obtained in the swollen state (see Figures 5-7), The thick fibrils are aligned parallel to the draw direction as determined from the shadowing direction (the shadowing was always done perpendicular to the draw direction).

The surface morphology of a region relaxed by the free-drying method is shown in Figure $14 \mathrm{a}$ and $\mathrm{b}$. It does not show any noticeable difference from that of the dried taut specimen; the same kind of micronecking features, lamella fragments and pulled-out fibrils, are observed. Moreover the pulled-out fibrils (thick ones) are still aligned almost parallel to the original draw direction with the thin fibrils lying at an angle to the thick ones. The area in Figure 14 a shown in a rectangle suggests that the short rodlike structure is produced by fragmentation of the long rod-like structure when the latter is

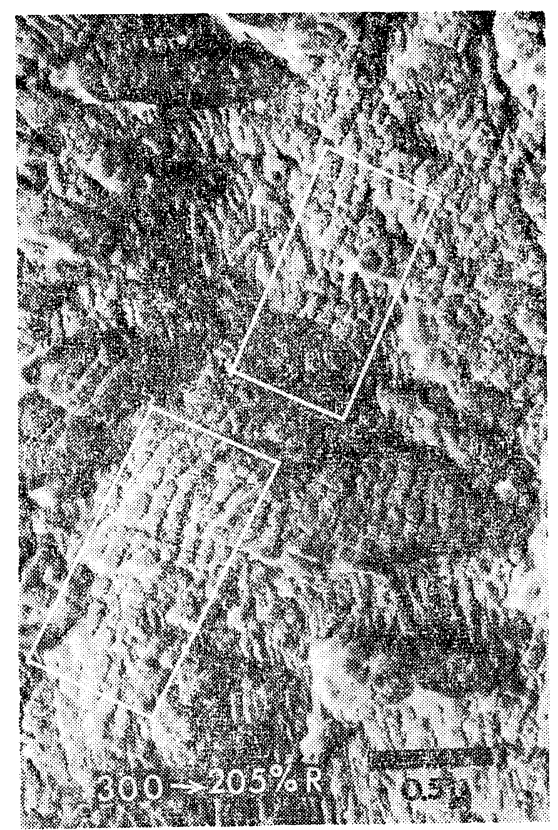

(a) subjected to a tensile shear stress.

The corresponding WAXD patterns of the dried taut specimen and the free-dried ones are shown in Figure 15. There is a superposition of a fiber pattern and Debye-Scherrer rings. Even from relatively highly stretched regions (amount of elongation is given in each photograph) there is some contribution from randomly oriented crystallities. This WAXD result agrees very well with the electron microscope observation in that they consist of fibers and fragments of lamellae.

Since the surface morphology of the dried taut specimen is very similar to that of the relaxed specimen and both are quite heterogeneous, it is hard to detect any morphological change before and after retraction without making close comparisons on the same selected area. The same area observations were made at various elongations in an attempt to determine which component is taking the major role in the retraction phenomenon. The results are shown in Figures 16-19 where the left side is the replica of the dried taut specimen and the right

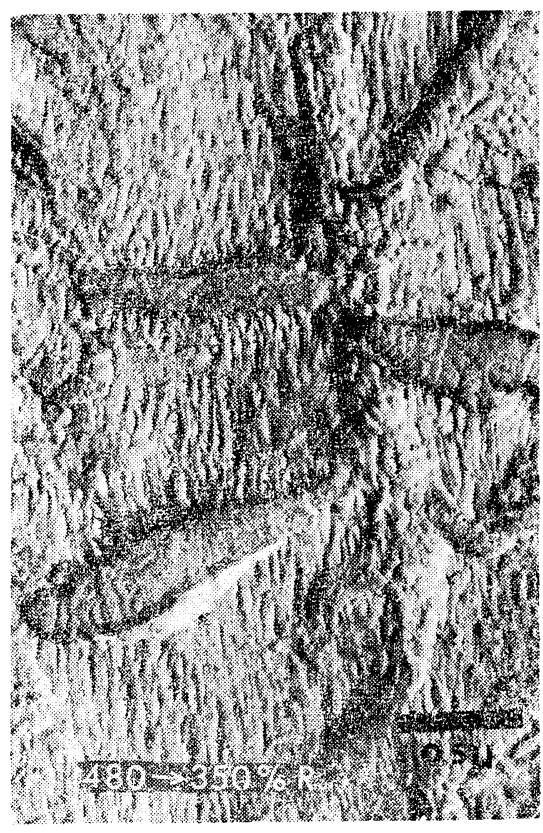

( b )

Figure 14. Electron micrographs of relaxed regions: (a) from 300-\% elongation to 205-\%, (b) $480-\%$ elongation to $350 \%$. The lamellae within the outlined regions in (a) appear to be breaking up, perhaps leading to the formation of the short rod-like structures. 

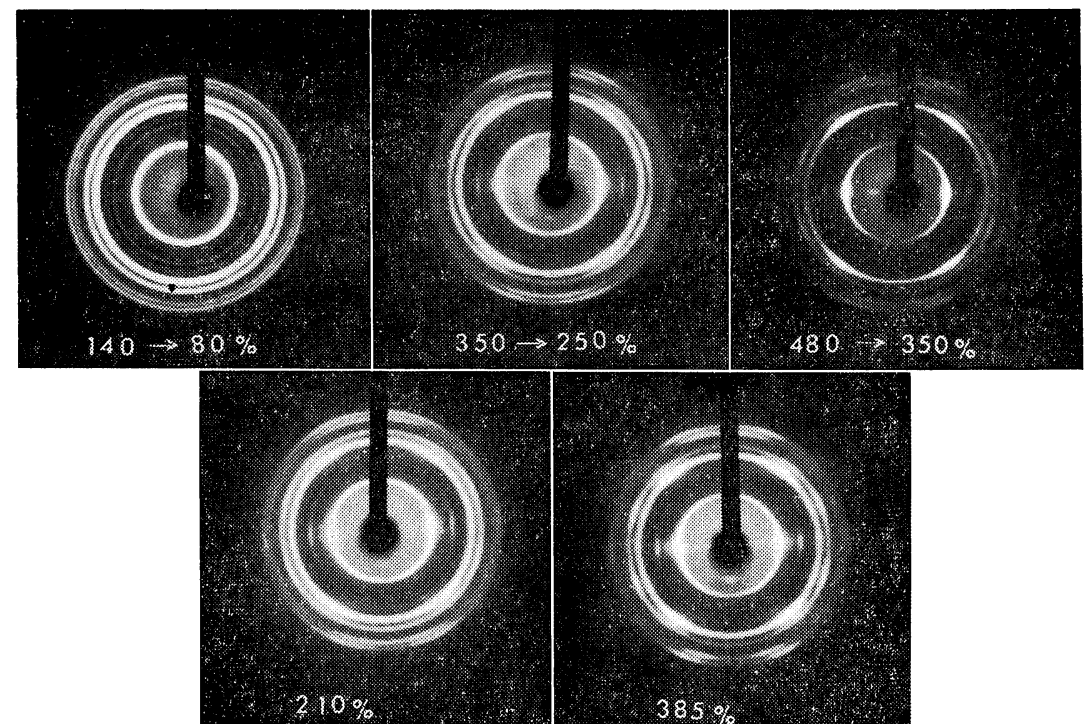

Figure 15. WAXD patterns of stretched and relaxed (upper) and dried taut (lower) specimens; elongations are given in each pattern.

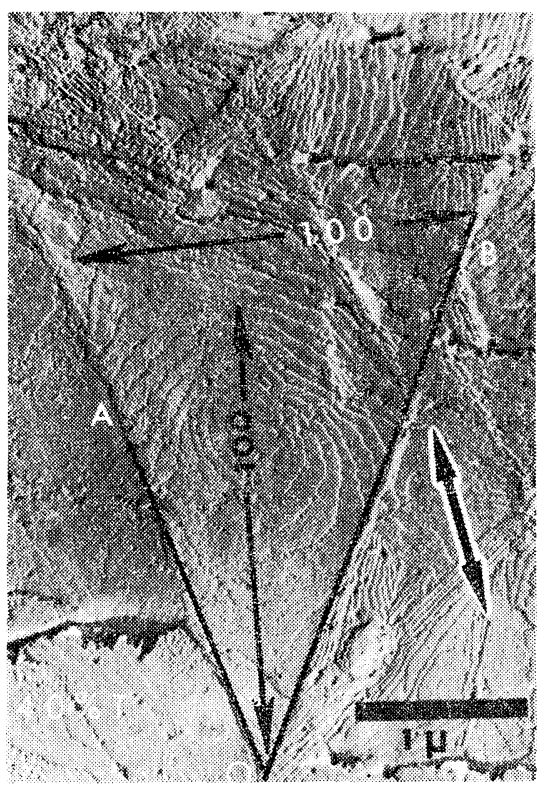

(a)

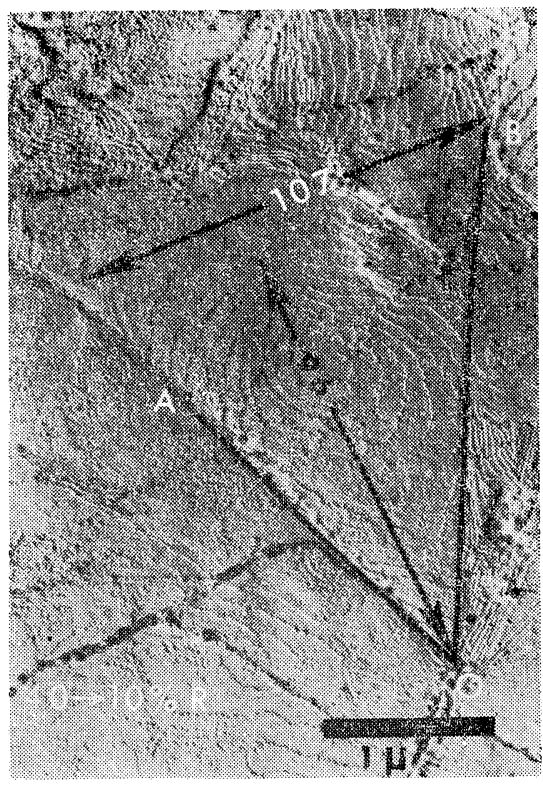

(b)

Figure 16. Electron micrographs of the same area of a sample initially drawn $40 \%$ before (a) and after (b) retraction caused by reswelling. Collodion 2 stage replica. 


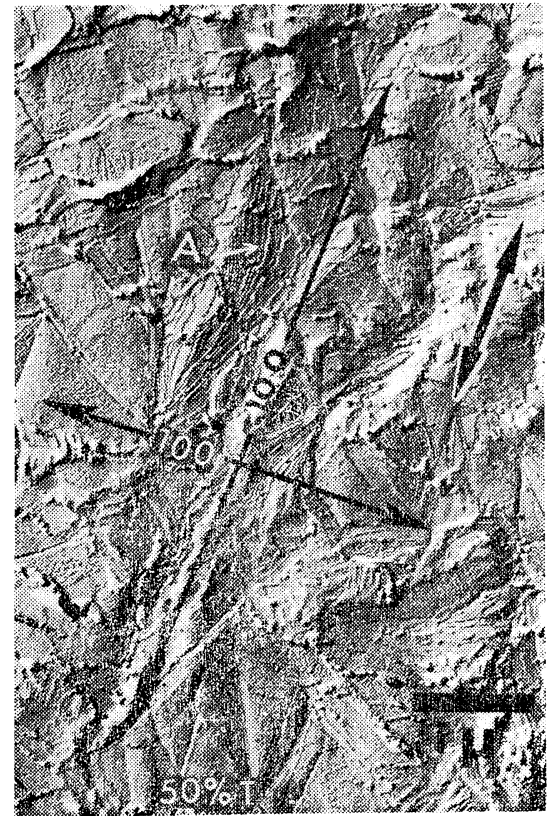

(a)

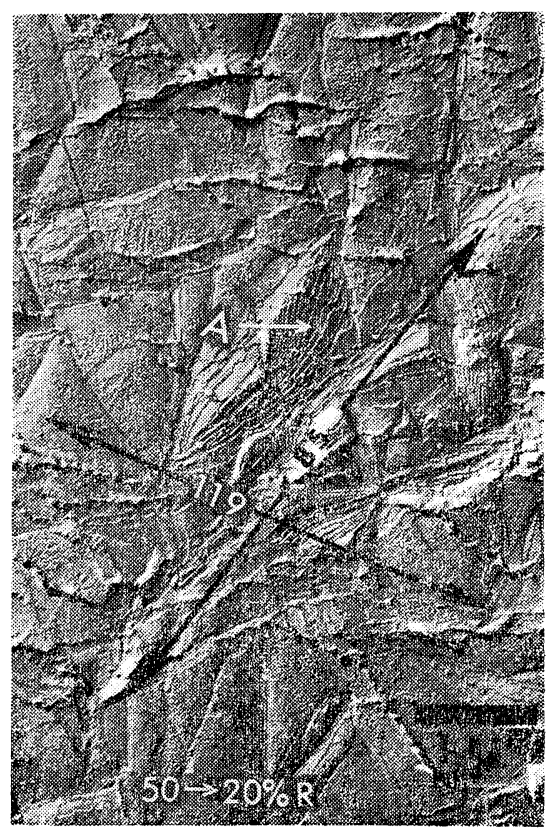

(b)

Figure 17. Electron micrographs of the same area of a sample initially drawn $50 \%$ before (a) and after (b) retraction caused by reswelling. Collodion 2 stage replica.

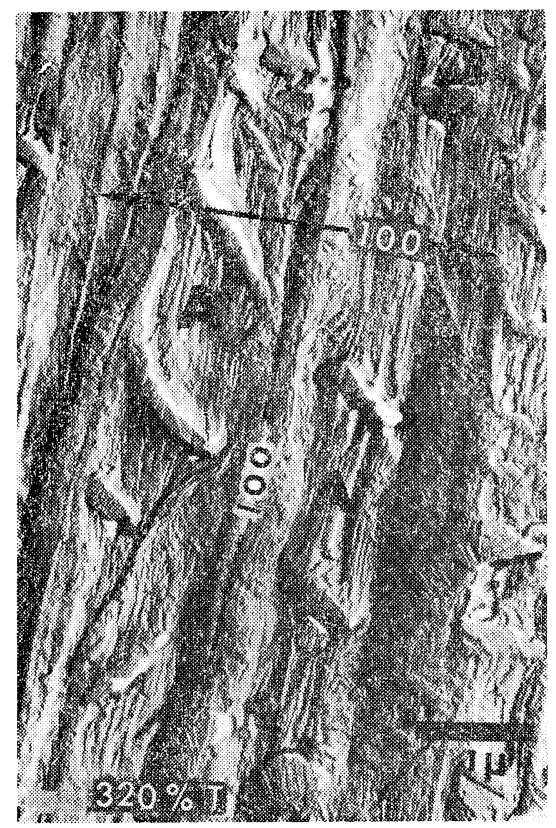

(a)

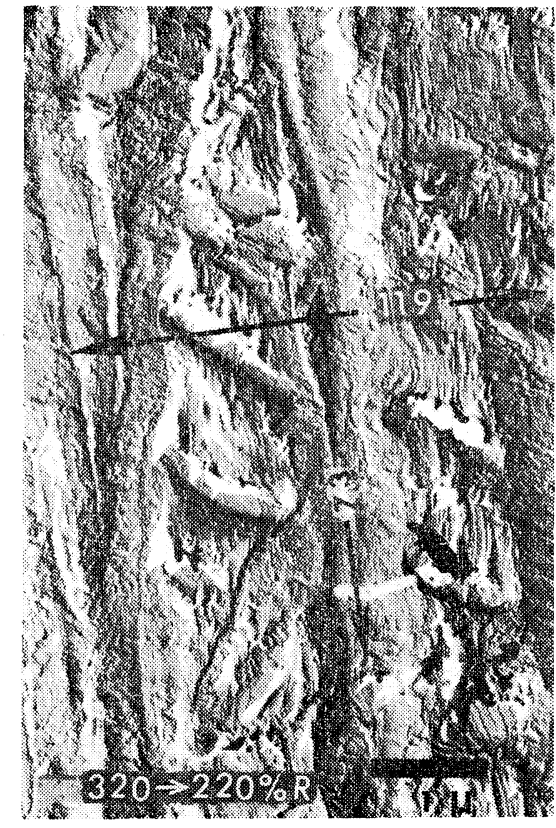

(b)

Figure 18. Electron micrographs of the same area of a sample initially drawn $320 \%$ before (a) and after (b) retraction caused by reswelling. Collodion 2 stage replica. 


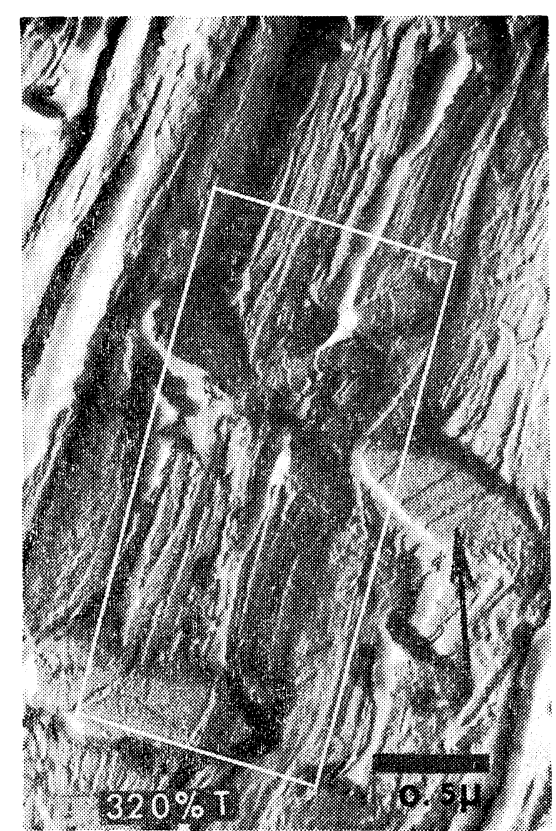

(a)

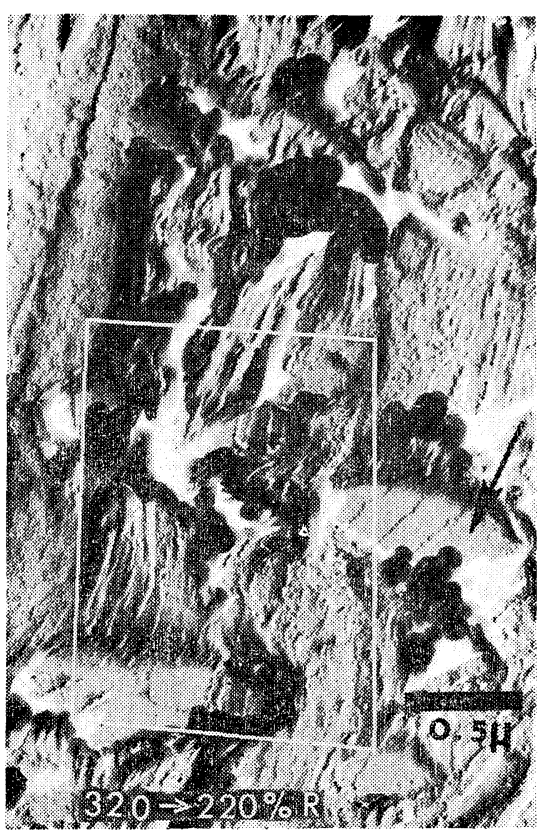

(b)

Figure 19. Similar electron micrographs as in Figure 18, under higher magnification, indicating no change in the lamellar fragments (arrow).

side is taken after it is reswollen and dried free. The amount of elongation is given in each photograph.

At low elongation, where few or no fibrils are observed (Figures 16 and 17), the main change during the retraction is a dimensional one. The distance between the pairs of points shown in the photographs, in arbitrary units, indicates that some retraction along the original drawing direction and some expansion perpendicular to it are taking place. This is clearly demonstrated in Figure 16 where the angle AOB of the triangularly shaped boundary of the spiral growth in the center of photograph has changed from $43^{\circ}$ to $49^{\circ}$ as it retracted. Also in Figure 17 the area marked A clearly demonstrates a dimensional change. Since no apparent fibrils are observed in Figure 16 and the marked area in Figure 17, it can be concluded that interlamellar slip is taking a significant role in the retraction, following small deformation and presumably in the original deformation also.

At higher elongation, where a fibril structure is predominant, the retraction takes place ex- clusively in the pulled-out fibril region. Although no individual fibrils can be seen on these same area replicas, it is reasonable to assume that the area marked $\mathrm{A}$ in Figure 18 consists of the same kind of fibrils as observed in Figures 13 and 14. As a result of shrinkage in the fibril regions, an overall retraction along the fibril direction and some expansion perpendicular to it are observed. Under higher magnification, shown in Figure 19, an upright rectangle shown in the photograph approaches to a square as the specimen retracted while the fragment of lamellae indicated by an arrow does not show any dimensional change.

No direct observation of the thickening of an individual fibril was possible; however it can be assumed that thickening of the fibrils is taking place during the retraction process similar to that observed in the case of the fibrils on the swollen fracture surface. It is also reasonable to assume that the overall expansion perpendicular to a fibril direction is caused by the thickening of the pulled-out fibrils. Although no fibrils are observed in the slightly drawn sam- 
ples, it may be that short ones exist or are formed between each lamella and it is these that retract during the apparent interlamellar slip.

\section{DISCUSSION AND CONCLUSIONS}

\section{Swelling}

The swelling of P4MP1 with various solvents appears to be reversible except for the fact that a small amount of low-molecular-weight material is extracted; the dimensions before swelling and after deswelling are the same and WAXD patterns before and after swelling (even the one taken in the swollen state) show no difference indicating that the agent is effective only in amorphous and/or interlamellar regions.

Bassett and Salovey ${ }^{10,11}$ have previously studied chemical reactions in mats of P4MP1 single crystals; they conclude that chemical agents are effective only in the interlamellar regions. For instance chlorination and oxidation of P4MP1 crystal mats, as shown by large increases in SAXD intensity, occur only at the fold surfaces. Bassett and Salovey also reported that simply moistening a single crystal film with carbon tetrachloride resulted in an intensification of the SAXD pattern indicating that the $\mathrm{CCl}_{4}$ swells the polymer by preferentially penetrating between the lamellae. They suggest bulk P4MP1 should show similar features (see below). However their samples would be expected to differ from our melt-crystallized samples in that one expects there to be fewer tie-molecules or interlamellae links in the mats and, consequently, less resistance against separation of the lamellae provided that the interior of the lamellae in both cases have the same perfection and degree of resistance to penetration of the chemical agents.

Woodward ${ }^{12}$ reported that the crystallinity of P4MP1 single crystal mats, estimated from the $\mathrm{X}$-ray diagram, was of the order of that found for annealed melt-crystallized specimen (65$70 \%$ ) indicating that the single crystal aggregates contain essentially the same amount of disordered regions as annealed melt-crystallized specimens. Takayanagi, et al.,${ }^{13}$ reported that the X-ray crystallinity of single crystal mats was $50-76 \%$, depending on preparation conditions, which was of the order of the crystallinity of the specimen crystallized slowly from the melt $(67 \%)$. With respect to the absolute value it should be pointed out that Karasz, et al. ${ }^{6}$ reported a much lower crystallinity for P4MP1 slowly crystallized from the melt $(28$ and $40 \%$, as estimated from calorimetry and X-ray diffractometry respectively). In our experience, $\mathrm{X}$-ray determinations of absolute crystallinity are subject to considerable uncertainty since the "amorphous scattering" is of nearly uniform intensity over a wide range of angles. Most of the amorphous material present must be attributed to an "amorphous" fold surface ${ }^{14}$ and interlamellar material. Any imperfections in the interior of the crystal are not sufficiently distorted as to be easily attacked by the swelling agent. If any swelling of defect regions within the crystal occurred, one would expect it to lead to further distortion of the crystal; however the WAXD pattern remained the same with respect to both lattice spacing and line width.

The WAXD data strongly suggests that one should be able to observe the interlamellar position of the swelling agent by means of SAXD; Descamps-Carlier, et al., ${ }^{5}$ were able to measure the increase in spacing for polyethylene swollen with biphenyl while Bassett and Salovey ${ }^{10,11}$ produced an increase in the SAXD intensity of a P4MP1 single crystal mat by introducing electron-dense atoms. Sincethe density of the crystalline phase of P4MP1 at room temperature is less than that of the amorphous phase ${ }^{7,15,16,17}$, introducing electrondense atoms into the amorphous region or between the lamellae should yield an increase in SAXD intensity at all levels of swelling. Our attempt to observe the location of the swelling agent in the melt-crystallized specimens by means of SAXD was unsuccessful. Although we also observed some intensification of SAXD in single crystal aggregates moistened with carbon tetrachloride, the melt-crystallized samples did not show any evidence of a peak in either the unswollen state or when swollen with benzene or carbon tetrachloride at room temperature.

Of interest with respect to SAXD studies of bulk polymers are the electron microscope observations of two lamellar thicknesses on the fracture surfaces. It has been reported that the smaller $\left(l_{2}\right)$ of the two spacings frequently ob- 
served for bulk polymers rather than the larger $\left(l_{1}\right)$ most closely agrees with the thickness of the lamellae observed on free surfaces. ${ }^{18}$ For our P4MP1 the lamellae at an angle to the fracture surface (i.e., the spacing of the striations as in Figures 6 and 7) are approximately 3 times as thick as those observed parallel to either fracture or free surfaces. Unfortunately, as indicated above, we were not able to obtain SAXD patterns from the bulk P4MP1 and thus cannot compare the SAXD and electron microscope results. These results do, however, tend to confirm the recent extensive study of POM by Burmester $^{19}$ in which similar observations were reported of two lamellar thicknesses on fracture surfaces, those at an angle to the surface being about twice as thick as those parallel to the surface. The thicker one corresponded to $l_{1}$, the thinner, which were similar to those on free surfaces, to $l_{2}$. Although one would expect the two thicknesses to be due to different aspects of the same structure, their relationship at this time is still unclear.

The separation of the lamellae (connected by interlamellar fibrils), although not observable by SAXD, was clearly observed by electron microscopy of the fracture surface of BCB swollen specimens (Figures 5-7). This is the first such observation of swollen samples. Previously two types of interlamellar links have been reported in the literature, both of which are formed during crystallization and are assumed to consist of ordered, aligned molecules $(2,3,20$ -22). Davis ${ }^{2}$ observed the separation of the lamellae, with fibrils drawn across the cracks, on the surface of an annealed, injection-molded POM subjected to a slight strain (15\%) parallel to the injection direction. The origin of this type of structure (oriented lamellae and fibrils) in injection-molded samples can be explained, for instance, by the mechanism discussed by Clark $^{3}$. It is similar to that proposed for the formation of "shishkebab" crystals under shear stress. ${ }^{23}$ According to Clark's paper, the oriented lamellae nucleated by shear produced fibrils occur only in the outer several mils of the sample. Presumably similar tie-links in the form of "Reneker webs" occur in the interior within and between spherulites. In our work, in which P4MP1 was crystallized from the melt pre- sumably without any fibril nuclei, "Reneker webs" could also be expected between parallel aligned lamellae. The fibrillinks connecting opposite surfaces of the cracks on the $\mathrm{BCB}$ swollen samples may result from the pulling out of the "Reneker webs" during the crystallization of the BCB. However it should be pointed out that the lamellae do not appear to be disturbed in the vicinity of the fibrils, even when a single lamellae has fibrils drawn from both surfaces.

The intercrystalline links proposed by Keith, Padden, and Vadimsky ${ }^{20-22}$ seem to be different from those of Reneker in that theirs are very long (up to a micron) and they assume the molecules in the intercrystalline links lie perpendicular to those in lamellae (see Figure 1 in ref 22) whereas in Reneker's case the molecules in the pulled-out fibrils presumably lie parallel to those in the lamellae. In addition Vadimsky, Keith, and Padden $^{24}$ reported that the original links usually showed no sign of reorganization upon annealing at $135^{\circ} \mathrm{C}$ while fibrils in highly drawn portions of a film developed striations transverse to the draw direction. The interlamellar links we observed in the swollen state, as shown in Figures 8 and 9, apparently reorganize upon deswelling since they shorten and become larger in diameter. Thus the pulled-out fibrils (thick ones) we observed would appear to be most closely related to those of Reneker except that the mechanism by which they are drawn out when the BCB crystallizes and enlarges certain interlamellar regions is not obvious from the model.

The thickening of the fibrils, both thick and thin, was observed upon retraction during deswelling. Although both fibrils behave almost identically upon deswelling, they seem to be different structural units; the distribution curves indicate two discrete maxima, 80 and $200 \AA$ in the swollen state and 90 and $220 \AA$ after deswelling and the thin ones lie at an angle to the thick ones. Thin fibrils may be produced by branching of the thick ones or further fibrillation of them, but the mechanism of formation of the thin fibrils still remains unexplained, especially for the ones lying at a right angle to the thick ones (see the arrows in Figure 7).

As indicated above another problem that remains unexplained is that there is no indication 
of void formation or disruption in the lamellae oriented normal to the fracture surface from which the numerous fibrils are pulled and that lamellar surfaces which are lying parallel to the fracture surface of BCB swollen specimen, when fractured both at liquid nitrogen temperature and at room temperature, showed no sign of pulled-out fibrils. For room temperature fracture, many bumps, probably resulting from beading of broken fibers, were observed on the edges of the lamellae oriented almost perpendicular to the surface. Although no complete satisfactory explanation could be found, the fact that no broken ends of fibrils were observed in the cracks, combined with the observation of rather smooth lamellar surfaces parallel to the fracture surface, suggests that drawing back of broken fibrils and an almost complete reorganization within the lamellae may be the case.

The above discussion is based on the assumption that the links are present in some form in the material when crystallized. It is also possible, however, particularly in view of the large amorphous content of even slowly crystallized polymer, that they are drawn out of the interlamellar "amorphous" material. The lamellar cohesion would then be due to cilia and tie molecules incorporated within the fibrils, retraction occurring either by relaxation within the fibrils or by a complete retraction and rerandomization of the fibrils. This would also be in agreement with the presence of smooth lamellar surfaces, sufficient molecular motion occurring to smooth out any irregularities in the amorphous surface layer.

\section{Deformation}

P4MP1 molded specimens are quite brittle unless they are swollen with various solvents. In previous work on crystalline polymers Hay and Keller $^{1}$ observed a large increase in ductility of a linear PE film when moistened with a solvent (o-chloro-phenol). McCann and Geacintov ${ }^{25}$ have observed large increases in elongation at break as well as drastic changes in other properties when P4MP1 was blended with various amounts of petroleum jelly. They ${ }^{25}$ claim that this is due to the decrease of internal friction of the polymer owing to the formation of uniform crystallites or small domain areas; as an explanation for the decrease of the yield strength of the blended samples, they claim that the crysstalline regions of the polymer become plasticized sufficiently to allow them to rearrange.

In our work, however, although the specimen swollen with benzene showed neither a plasticization effect in the crystalline region nor the formation of smaller crystallities, it underwent macroscopically uniform deformation when drawn either in air or in the swelling agent. Elongations of up to 480 and 200\%, when drawn in air or the swelling agent, respectively, could be obtained. On a microscopic level, the large scale deformation was inhomogeneous in nature, fibrils and lamellae being observed. At low elongation (up to about 50\%), lamellar slip as well as fibril formation occur (Figure 16).

The characteristic feature of the deformation process of the swollen material is a micronecking process, the number and size of the micronecks increasing with increasing elongation. It has previously been reported by Woodward and Morrow $^{26}$ that single crystals of P4MP1, when stretched on a Mylar substrate, undergo micronecking. Cracks, spanned by fibrils, and a mosaic structure form due to the result of cooperative micronecking and slip; at as low as $40-\%$ elongation in any direction with respect to the crystallographic axis of the crystal, many cracks and accompanying fibrils are observed. This is in contrast to polyethylene single crystals which could be elongated along the a axis up to $150 \%$ without molecular unfolding or fracture. ${ }^{27}$ It has been suggested ${ }^{13,28}$ that P4MP1 single crystal and melt-crystallized samples consist of a mosaic block structure resulting from the coordinated association of helices, each mosaic block being composed of like-handed helices. Although the size of the mosaic blocks proposed by the various investigators is quite different $(0.5-1 \mu$ by Morrow, et al. ${ }^{28}$ from annealing experiments and 34- $160 \AA$ by Takayanagi, et al. ${ }^{13}$ from viscoelastic measurements), the micronecking of P4MP1 may possibly be attributed to the existence of the mosaic blocks leading to numerous weak points within the crystals.

Upon shrinkage two different types of morphological change were observed reversing the effects of the initial strain; at low elongation inter-lamellar slip mainly took place, while shrinkage of the fibrils was predominant at high elonga- 
Morphology and Deformation of Swollen P4MP1. I.

tion. The latter appears to be the same retraction phenomena as that observed upon deswelling of BCB swollen specimens, Similar phenomena have been observed during the thermal shrinkage of partially drawn POM film. ${ }^{29}$ Of interest here are the several types of fibrils observed in POM and their behavior during shrinkage. Type 1 were very short fibrils seen at the edges of lamellae oriented parallel to the surface that disappeared during heat shrinkage. Their retraction may accompany (and cause) interlamellar slip. Although possibly similar fibrils may be seen on the edges of the spiral growth in Figure 16 they seem to remain during shrinkage of the P4MP1. Types 2 and 3 were short fibrils, some of which appeared to thicken during shrinkage and remain as an independent, smooth fiber. Type 4 were long fibrils, apparently similar to those observed in fully drawn polymers, which developed striations normal to the draw direction when annealed. Their structure and behavior can be interpreted, to an extent, in terms of the "wavy lamella" structure proposed by Bonart. ${ }^{30}$

The relative shrinkage in P4MP1 stayed almost constant above 130-\% elongation, indicating that the amount of shrinkable material increases directly with elongation and that its retractive behavior remains constant above 130-\% elongation. This is not the case for thermal shrinkage of drawn $\mathrm{POM}^{29}$ and drawn $\mathrm{PE}^{31}$; for these polymers it was observed that the relative shrinkage reaches a maximum at a certain elongation, i.e., at $140 \%$ for POM and 100\% for PE.

The observations reported here and for $\mathrm{POM}^{29}$ suggest at least two types of fibril structure. Although a "wavy lamella" model appears to reasonably well describe the structure of the type usually seen in fully drawn polymers, the structure of the fibrils observed here for P4MP1 and the types 2 and 3 in POM $^{29}$ would appear to have a different and, as yet, unknown structure. They frequently appear to be more individual, with little or no lateral cohesion other than the interconnecting "thin" fibrils. The P4MP1 observations suggest they may be related to or at least derived from the fibril-links observed in swollen P4MP1. Their formation is not peculiar to the micronecking process, however, since POM type 4 as well as those observed stretched across cracks in PE crystals ${ }^{32}$ both of which form by a micronecking process develop striations during annealing.

Since the original structure of the P4MP1 fibrils is not known, the mechanism of shrinkage is also not known at present; it must satisfy the following observations for thermal and solventinduced shrinkage.

(1) The WAXD patterns taken after retraction are almost identical to ones taken from a specimen stretched directly to the final elongation (P4MP1).

(2) The fibrils retain their alignment and possibly become slightly thicker as well as shorter (P4MP1 and POM).

(3) Upon annealing the fibrils do not develop striations.

Direct examination of the fibrils by dark-field microscopy would be desirable but has not been possible to date.

Acknowledgment. Appreciation is expressed to Teijin Limited and the National Science Foundation for financial support for this research.

\section{REFERENCES}

1. I. L. Hay and A. Keller, Kolloid-Z., 204, 43 (1965).

2. H. A. Davis, J. Polym. Sci., Part A-2, 4, 1009 (1966).

3. E. S. Clark, SPE Journal, 23, 46 (1967).

4. P. Mears, "Polymers: Structure and Bulk Properties", Van Nostrand Co. Ltd., London, 1965, Chapter 12.

5. Par. J. Descamps-Carlier, G. A. Homes, and J. J. Point, Makromol. Chem., 109, 1 (1967).

6. F. E. Karasz, H. E. Bair, and J. M. O'Reilly, Polymer, 8, 547 (1967).

7. J. H. Griffith, and B. G. Rånby, J. Polym. Sci., 44, 369 (1960).

8. Y. Hase, and P. H. Geil, Polymer $J, 2,579$ (1971).

9. G. Natta, Makromol. Chem., 35, 94 (1960).

10. D. C. Bassett, and R. Salovery, J. Polym. Sci., Part B, 2, 503 (1964).

11. D. C. Bassett, Polymer, 5, 457 (1964).

12. A. E. Woodward, Polymer, 5, 293 (1964).

13. M. Takayanagi, and N. Kawasaki, J. Macromol. Sci.-Phys., B1 (4), 741 (1967).

14. E. W. Fischer, and F. Kloos, J. Polym. Sci., in press.

15. F. C. Frank, A. Keller, and A. O'Conner, Phil. Mag., 4, 200 (1959). 
16. B. G. Ranby, K. S. Chan, and H. J. Brunterger, J. Polym. Sci., 58, 545 (1962).

17. M. Litt, J. Polym. Sci., Part A, 1, 2219 (1963).

18. R. G. Brown, and R. K. Eby, J. Appl. Phys., 35, 1156 (1964).

19. A. Burmester, Ph. D. Thesis, Case Western Reserve University, Cleveland, Ohio, 1970.

20. H. D. Keith, F. J. Padden, Jr., and R. G. Vadimsky, Science, 150, 1026 (1965).

21. H. D. Keith, F. J. Padden, Jr., and R. G. Vadimsky, J. Polym. Sci., Part A-2, 4, 267 (1966).

22. H. D. Keith, F. J. Padden, Jr., and R. G. Vadimsky, J. Appl. Phys., 37, 4027 (1966).

23. A. J. Pennings, and A. M. Kiel, Kolloid-Z., 205, 160 (1965).

24. R. G. Vadimsky, H. D. Keith, and F. J. Padden,
Jr., J. Polym. Sci., Part A-2, 7, 1367 (1969).

25. J. McCann, and C. Geacintov, J. Appl. Polym. Sci., 13, 2383 (1969).

26. A. E. Woodward, and D. R. Morrow, J. Polym. Sci., Part A-2, 7, 1651 (1969).

27. P. H. Geil, J. Polym. Sci., Part A, 2, 3813 (1964).

28. D. R. Morrow, G. C. Richardson, L. Kleinman, and A. E. Woodward, J. Polym. Sci., Part A-2, 5, 496 (1967).

29. A. Siegmann, to be published in J. Macromol. Sci.-Phys.

30. R. Bonart, as presented by Stuart H. A. in Ann. N.Y. Acad. Sci., 83, 3 (1959).

31. S. Kinoshita, and T. Takizawa, Kobunshi Kagaku (Chem. High Polymers, 19, 397 (1962).

32. P. H. Geil, J. Polym. Sci., Part A, 2, 3835 (1964). 